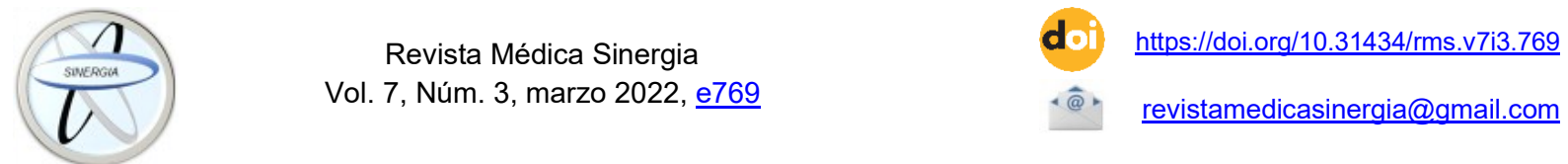

\title{
Fórmulas y marcadores endógenos para la evaluación de la función renal en adultos mayores
}

Equations and endogenous markers for the assessment of renal function in the elderly

\author{
${ }^{1}$ Dr. Dany Montero Valverde \\ Hospital Manuel Mora Valverde - Caja Costarricense de Seguro Social (CCSS), \\ Puntarenas, Costa Rica \\ https://orcid.org/0000-0003-0752-7756 \\ ${ }^{2}$ Dr. Ignacio Abarca Brenes \\ Hospital Manuel Mora Valverde - Caja Costarricense de Seguro Social (CCSS), \\ Puntarenas, Costa Rica \\ https://orcid.org/0000-0002-3050-3480
}

${ }^{3}$ Dra. Amanda Chavarría Rojas

Hospital Calderón Guardia - Caja Costarricense de Seguro Social (CCSS),

San José, Costa Rica

https://orcid.org/0000-0001-9125-3211

Recibido 26/01/2022
Corregido
$03 / 02 / 2022$
Aceptado

$10 / 02 / 2022$

\section{RESUMEN}

La tasa de filtración glomerular (TFG) es el producto del número de nefronas multiplicado por la tasa de filtración glomerular media de una sola nefrona. Esta medida en el adulto mayor, está condicionada por una serie de factores que conllevan al diagnóstico erróneo de la enfermedad renal, sin tomar en cuenta los cambios que sufre el riñón con el envejecimiento. El uso de diferentes marcadores endógenos como la creatinina está sujeta a la disminución de la masa muscular y la dieta, sin embargo, su facilidad de medición, su bajo costo y la amplia disponibilidad de análisis, la convierten en uno de los más utilizados en la práctica clínica. La cistatina $\mathrm{C}$ no se afectada por factores como la edad, el sexo, la raza y la masa muscular, por lo que se considera un mejor biomarcador en pacientes geriátricos, los cuales presentan mucha variabilidad en su composición muscular. Actualmente la fórmula Colaboración sobre Epidemiología de la Enfermedad Renal Crónica (CKD-EPI) es la recomendada por las últimas guías Kidney Disease Improving Global Outcomes (KDIGO) del 2012 para estimar el filtrado glomerular. Además, se han desarrollado otras ecuaciones CKD-EPI con cistatina $C$ y CKDEPI con creatinina-cistatina. 
PALABRAS CLAVE: tasa de filtración glomerular; enfermedad renal; creatinina; cistatina.

\section{ABSTRACT}

The glomerular filtration rate (GFR) is the product of the number of nephrons multiplied by the mean glomerular filtration rate of a single nephron. This measure in the elderly is conditioned by a series of factors that lead to the erroneous diagnosis of kidney disease, without taking into account the changes that the kidney undergoes with aging. The use of different endogenous markers such as creatinine is subject to the decrease in muscle mass and diet, however, its ease of measurement, its low cost and the wide availability of analysis, make it one of the most widely used in the clinical practice. Cystatin $C$ is not affected by factors such as age, sex, race and muscle mass, so it is considered a better biomarker in geriatric patients, who have a great variability in their muscle composition. Currently, the Chronic Kidney Disease Epidemiology Collaboration (CKD-EPI) formula is recommended by the latest Kidney Disease Improving Global Outcomes (KDIGO) guidelines of 2012 to estimate glomerular filtration rate. In addition, other CKD-EPI equations with cystatin C and CKD-EPI with creatinine-cystatin have been developed.

KEYWORDS: glomerular filtration rate; kidney disease; creatinine; cystatin

${ }^{1}$ Médico especialista en Geriatría y Gerontología, graduado de la Universidad de Hispanoamericana (UH). Cód. MED11130. Correo: dannymonterov0409@gmail.com

${ }^{2}$ Médico general, graduado de la Universidad de Ciencias Médicas (UCIMED). Cód. MED16518. Correo: igabarca95@gmail.com

${ }^{3}$ Médico especialista en Medicina Interna, graduada de la Universidad Latina de Costa Rica (U. Latina). Cód. MED14075. Correo:

amachavarria20@gmail.com

\section{INTRODUCCIÓN}

Actualmente, la población mundial se encuentra en un proceso de envejecimiento, es por esta razón que debemos prepararnos para encontrar la mejor forma de abordar las patologías más frecuentes que sufren los adultos mayores.

Debe señalarse que el filtrado glomerular disminuye hasta $8-10 \mathrm{ml} / \mathrm{min} / 1,73 \mathrm{~m} 2$ por década a partir de los 30-40 años de vida, por lo que este método por si solo no es un buen parámetro para definir la enfermedad renal. Esto conduce muchas veces a diagnósticos, que posteriormente, limitan el abordaje terapéutico en muchos adultos mayores. El corte de tasa de filtración glomerular (TFG) menor a $60 \mathrm{ml} / \mathrm{min} / 1,73$ $\mathrm{m} 2$ se ha asociado a mayor morbilidad cardiovascular, aumento de la mortalidad y aparición de enfermedad renal terminal. No queda claro en qué circunstancias los adultos mayores no hipertensos, no diabéticos, ni portadores de otras enfermedades asociadas, tendrán una TFG menor este corte, condicionando a un diagnóstico y pronóstico menos favorable a futuro.

El objetivo de esta revisión es esclarecer cual de las fórmulas más conocidas para la estimación de la TFG es la más recomendada para ser aplicada en el paciente adulto mayor, así como el marcador endógeno más adecuado para evaluar la función renal de este grupo etario.

\section{MÉTODO}

Se realizó una revisión bibliográfica de los principales artículos y estudios científicos relevantes para el objetivo de este artículo. Se buscaron estudios en los idiomas ingles y español, así como publicaciones entre los años 2016 y 2021 por medio de base de 
datos médicas como Pubmed, Cochrane Library y Cinhal. Las palabras de búsqueda utilizadas fueron "filtración glomerular", "enfermedad renal", "creatinina" y "cistatina". Para la selección de estos artículos, se leyó cada uno de forma completa y se seleccionó la información mas relevante de cada uno para desarrollar esta revisión.

\section{ESTIMACIÓN DE LA TASA DE FILTRACIÓN GLOMERULAR}

El proceso fisiológico de crear un ultrafiltrado de sangre a medida que esta fluye a través de los capilares glomerulares hacia la cápsula de Bowman por unidad de tiempo se conoce como tasa de filtración glomerular. Inicialmente, la TFG es el producto del número de nefronas multiplicado por la tasa de filtración glomerular media de una sola nefrona. Entre sus determinantes, se incluyen factores hemodinámicos dentro de la red capilar glomerular y las propiedades de la pared capilar (1-3). Numerosas afecciones fisiológicas y patológicas causan variabilidades entre distintos individuo, incluidas la hora del día, la ingesta de proteínas en la dieta, el ejercicio físico, la edad, el embarazo, la composición corporal, la hiperglucemia, el uso de fármacos antihipertensivos, el exceso o déficit de líquido extracelular, así como una lesión renal aguda o enfermedad crónica ya establecida. Todo lo anterior se suma a los cambios estructurales, funcionales $y$ moleculares propios del envejecimiento, por lo que se debe ser aún más cuidadosos con el cálculo de la TFG en esta población (1-3). El gold strandard para esta medición es utilizar el aclaramiento urinario de inulina, un método descrito por el fisiólogo estadounidense Homer Smith; sin embargo, sus principales limitantes son su costo, su dificultad para disolverse y mantenerse en una solución, así como un suministro muy limitado $(2,3)$.

- Estimación de la TFG con marcadores endógenos sérico: en la práctica clínica actual, este es el método más utilizado para el cálculo de la TFG aproximada de un individuo; esta se realiza de forma rutinaria cuando se desea dosificar un medicamento según la función renal. Se utiliza la cuantificación de los niveles séricos de los marcadores de filtración endógenos, sin necesidad de calcular el aclaramiento renal $(1,2)$.

Al realizar esta medición, se debe tener en cuenta que así como existen determinantes de la TFG, tales como la presión hidrostática glomerular, la presión coloidosmótica glomerular y la presión en la cápsula de Bowman, existen los no determinantes de la TFG como la dieta y la eliminación extrarrenal (intestinal y hepática) que va a influir y alterar la precisión a la hora de estimar la filtración glomerular (2).

Las ecuaciones de estimación toman en cuenta variables demográficas y clínicas (la edad, la raza, la presencia o no de otras enfermedades) como sustitutos de los no determinantes de la filtración glomerular que no son medidos. Estas ecuaciones proporcionan una estimación de la TFG más precisa que la concentración plasmática sola, sin embargo, debe comprenderse que no son exactas y deben interpretarse bajo esa condición $(2,4)$.

\section{MARCADORES}

\section{Marcador exógeno}

Se consideran como un marcador exógeno ideal a aquellas moléculas que son filtradas libremente en los glomérulos renales, 
excretándose por completo en la orina, sin ser reabsorbidas ni secretadas por los túbulos. Característicamente, estas deben tener un peso molecular inferior a $20 \mathrm{kDa}$ y que no se unan a las proteínas plasmáticas. Como se mencionó anteriormente, la inulina sigue siendo el gold standard para su uso en las mediciones de aclaramiento; otros marcadores exógenos son el iotalamato, el iohexol, el ácido etilendiaminotetraacético y el ácido dietilentriaminopentaacético, que a menudo son quelados con radioisótopos para facilitar su detección $(5,6)$.

\section{Marcadores endógenos}

Entre los marcadores endógenos, contamos con la creatinina, urea y cistatina $\mathrm{C}$. Estos suelen filtrarse en los glomérulos, pero presentan secreción o reabsorción tubular. Para evaluarlos, se puede comparar su aclaramiento urinario con el filtrado glomerular utilizando marcadores exógenos; y para saber su eliminación extrarrenal se compara su tasa de excreción urinaria con su tasa de generación $(5,6)$ :

- Creatinina: se identificó por primera vez en el año 1847, y se propuso como marcador de filtración glomerular en 1926. Esta se deriva del metabolismo de la fosfocreatina presente en el músculo, así como de la ingerida por consumo de carnes o suplementos de creatina.

La producción de creatinina es proporcional a la masa muscular de cada individuo, por lo cual representa un inconveniente para ser utilizada con adultos mayores, ya que la mayoría posee menor cantidad de músculo y pone en duda su utilidad clínica en esta población. Pequeñas elevaciones en la creatinina sérica de los adultos mayores reflejan un descenso del filtrado glomerular más significativo que lo que representarían en individuos jóvenes $(7,8)$. Parece ser claro que este marcador endógeno no es el más adecuado para calcular la TFG en el adulto mayor, sin embargo, por su facilidad de medición, su bajo costo y la amplia disponibilidad de análisis, es de los más utilizados en la práctica clínica (8).

- Cistatina C: esta molécula se identificó por primera vez en el año 1979, fue propuesta como marcador de filtración glomerular en 1985. Es una proteína de 122 aminoácidos, tiene un tamaño de 13 $\mathrm{kDa}$, es producida por todas las células nucleadas humanas y se encuentra distribuida por todo el líquido extracelular. Se reabsorbe en el túbulo proximal en un 99\%, donde es catabolizada completamente por las células tubulares y no retorna hacia el torrente sanguíneo (9). La independencia de la cistatina $\mathrm{C}$ de factores como la edad, el sexo, la raza y la masa muscular hace que esta sea un mejor biomarcador en pacientes geriátricos y pediátricos, los cuales presentan mucha variabilidad en su composición muscular. Entre los factores que pueden alterar la estimación de la TFG utilizando la cistatina $C$, se pueden señalar el fumado, la inflamación, el grado de adiposidad, enfermedad tiroidea y algunas neoplasias malignas $(1,9,10)$.

Desde el punto de vista de salud pública y epidemiológico, se ha demostrado que utilizar la cistatina $\mathrm{C}$ como complemento de la creatinina sérica permite estimar el riesgo con mayor precisión, así como ayudar a brindar un diagnóstico más claro a los pacientes con deterioro leve a moderado de la TFG. La mayor desventaja con respecto a este marcador es su costo, el cual puede ser 12 veces mayor que el de la creatinina $(9,10)$. 


\section{USO DE CREATININA SÉRICA O CISTATINA C}

Todas las ecuaciones están limitadas por la variabilidad de los determinantes no relacionados con la TFG, ya que esto ocasiona cambios en la concentración plasmática de los marcadores. Dado a esto, se debe tener presente factores como la generación a nivel celular, el consumo por la dieta, la secreción y la reabsorción en los túbulos renales, y la eliminación extrarrenal como en hígado o intestino.

Ninguna de estas ecuaciones funcionará bien en personas con variación importante de la producción de creatinina, por ejemplo, amputados de extremidades, individuos muy pequeños o grandes, personas con afecciones de desgaste muscular, ingesta alta o baja de carnes en la dieta. Se debe tomar en cuenta que la dieta cambia según las diferencias étnicas, así como también la composición corporal varía entre grupos raciales, es por esto que es poco probable que las ecuaciones desarrolladas sean precisas en poblaciones multiétnicas $(5,6)$.

Decir que la tasa de filtración glomerular medida con cistatina $\mathrm{C}$ es más precisa que la calculada con creatinina sérica no es correcto, puesto que se ha demostrado que la combinación de ambos marcadores da como resultado una estimación más precisa en poblaciones con diagnóstico o sin diagnóstico de enfermedad renal $(5,6,9)$.

\section{FÓRMULAS MÁS UTILIZADAS PARA CALCULAR LA TASA DE FILTRACIÓN GLOMERULAR}

Las ecuaciones como la de Cockcroft-Gault, MDRD (Modificación de la Dieta en la Enfermedad Renal) y CKD-EPI usan los marcadores endógenos como la creatinina sérica y la cistatina $C$ para hacer su respectiva estimación de la TFG.
Esto no contempla los cambios que suceden en la población adulta mayor. Utilizar marcadores exógenos como la inulina es más laborioso y costoso, lo cual limita su uso $(6,7)$. Entre las ecuaciones basadas en creatinina sérica, se ha buscado realizar una estandarización de las mismas, incluyendo la edad, el sexo, la raza y el tamaño corporal como sustitutos de la producción de creatinina marcada, por alto consumo de proteína en la dieta y masa muscular; a pesar de esto, estas fórmulas presentan sesgo entre una y otra población haciéndolas imprecisas.

\section{Ecuación de Cockcroft-Gault}

Esta fórmula utiliza la edad, el peso, el sexo y el nivel de creatinina sérica para el cálculo del aclaramiento de creatinina en $\mathrm{ml} / \mathrm{min}$. Fue desarrollada en 1973 por Donald W. Cockcroft junto con Matthew H. Gault en el servicio de nefrología del Queen Mary Veteran's Hospital en Montreal, Canadá; y publicada posteriormente en 1976. Fue formulada a través un ensayo de picrato alcalino con desproteinización, donde la población en estudio incluyó a 249 hombres estadounidenses blancos de entre 18-92 años de edad con un aclaramiento de creatinina medio de $73 \mathrm{ml} / \mathrm{min}$. Se realizó un factor de ajuste para las mujeres, basado en un $15 \%$ menos de generación de creatinina debido a la menor masa muscular de este grupo (1,11). Entre sus principales desventajas y limitaciones es que calcula el aclaramiento de creatinina en lugar del filtrado glomerular. Esto se convierte en un problema cuando se utilizan ensayos de creatinina sérica estandarizados, ya que sobrestima la TFG; sin embargo, en pacientes mayores de 80 años exagera el descenso de la TFG $(1,11)$.

Al incluir el peso del individuo, puede subestimar el aclaramiento de creatinina en 
personas obesas o edematosas, y no incluye variable de raza, por lo que suele subestimar la TFG en afroamericanos. Al utilizar métodos de ensayo más antiguos, se dificulta su calibración con métodos de ensayo más nuevos (3).

\section{Ecuación del estudio MDRD}

Esta fórmula fue desarrollada en el año 1999, e incluyó a 1070 hombres y mujeres

estadounidenses previamente diagnosticados con enfermedad renal crónica (ERC), con una TFG media de 40 $\left.\mathrm{ml} / \mathrm{min} / 1,73 \mathrm{~m}^{2}\right)$. En este estudio se observó los efectos de la restricción de proteína en la dieta y el control estricto de la presión arterial en la progresión de la $\operatorname{ERC}(1,3)$.

Esta fórmula buscaba mejorar la precisión de la Cockcroft-Gault, además de que estima la TFG y no el aclaramiento de creatinina. La ecuación incluye la superficie corporal en $\mathrm{ml} / \mathrm{min} / 1,73 \mathrm{~m}^{2}(1,4)$.

Inicialmente contaba con 6 variables, es por esto que se le llamaba MDRD-6, sin embargo un año después se publica la MDRD-4, una forma abreviada que incluía como variables la edad, el sexo, la raza y la creatinina sérica, excluyendo a la urea y la albúmina $(3,11)$.

En el año 2006, posterior a otro estudio de evaluación, resultó ser más precisa y con un menor sesgo que la ecuación CockcroftGault (3). Su principal limitante es que no se incluyó en ninguno de los estudios a personas sin enfermedad renal, personas diabéticas en tratamiento con insulina, personas menores de 18 años y mayores de 70 años, ni tampoco mujeres embarazadas. Por lo tanto, esta fórmula no es aplicable en la población general, ya que puede subestimar sistemáticamente la TFG medida a una TFG estimada más alta, de modo que no se pueden informar valores numéricos para niveles de TFG estimada $>60$ $\mathrm{ml} / \mathrm{min} / 1,73 \mathrm{~m}^{2}$. La otra variable a tomar en cuenta es la raza, ya que solo nombra a afroamericanos, blancos y otros, por lo que puede estar más sesgada en poblaciones de ciertas razas $(1,3,11)$.

\section{Ecuación CKD-EPI}

El Instituto Nacional de la Diabetes y las Enfermedades Digestivas y Renales (NIDDK) creó en el año 2009 la fórmula CKD-EPI (Colaboración sobre Epidemiología de la Enfermedad Renal Crónica), cuyo objetivo era superar las deficiencias de la ecuación del estudio MDRD del año 2006. Se llevó acabo con una población de 8254 individuos agrupados en 10 estudios a los cuales se les midió el filtrado glomerular mediante el aclaramiento urinario de iotalamato, con una TFG media de $68 \mathrm{ml} / \mathrm{min} / 1,73 \mathrm{~m}^{2}$; e incorporó variables como la edad, el sexo, la raza y los valores de creatinina plasmática del individuo (CKDEPI-creatinina) $(3,12,13)$. Esta incluyó individuos con y sin enfermedad renal, diabéticos, además de receptores de trasplantes de órganos y donantes potenciales; sin embargo, el estado de diabetes, el estado de trasplante de órganos y el peso no mejoraron el rendimiento de la ecuación. Al igual que con la ecuación del estudio MDRD, la variable raza incluye coeficientes solo para afroamericanos frente a blancos y otros $(1,4,14)$.

Según el estudio de validación externa en 3896 sujetos agrupados en otros 16 estudios, la comparación de CKD-EPI frente a MDRD pone en manifiesto que la primera brinda mejores resultados, en especial para valores de TFG>60 $\mathrm{ml} / \mathrm{min} / 1,73 \quad \mathrm{~m}^{2}$, disminuyendo su imprecisión frente a la medida directa del filtrado glomerular. Dado a esto, llegan a la conclusión que la CKD- 
EPI debería sustituir la MDRD en la práctica clínica $(1,3,4,11)$.

Esta es la ecuación recomendada por las últimas guías Kidney Disease Improving Global Outcomes (KDIGO) del 2012 para estimar el filtrado glomerular. También se han desarrollado otras ecuaciones CKD-EPI con cistatina $\mathrm{C}$ y CKD-EPI con creatininacistatina. $(1,3,4,11)$.

Entre sus limitaciones importantes se encuentran las asociadas con los niveles de creatinina sérica, la representación de la población, y en particular que no se cuenta con una muestra significativa de personas mayores de 70 años (el 15\% de la población tenía más de 65 años y el $28 \%$ eran diabéticos). Cabe mencionar que este estudio utilizó valores de creatinina sérica calibrados a la creatinina estandarizada, procedimiento que no se realiza con frecuencia en todo el mundo, hecho que hace que su aplicación externa sea poco confiable $(1,3,4,15)$.

\section{CONCLUSIÓN}

La enfermedad renal es una de las principales comorbilidades que afectan a los adultos mayores a nivel mundial. Este diagnóstico conlleva a repercusiones desde el punto de vista de salud pública y a limitaciones terapéuticas en muchos pacientes mayores de 65 años de edad. Debe quedar claro que la disminución de la tasa de filtración glomerular después de la tercera y cuarta década de vida es considerada un cambio normal del riñón durante el envejecimiento. Muchas veces, estos pacientes son diagnosticados con esta patología tan solo tomando en cuenta la medición del filtrado glomerular y utilizando marcadores endógenos cuya concentración y medición está sesgada en poblaciones de edad avanzada por variantes como el peso, el sexo, la raza y otras enfermedades concomitantes.

Es importante educar a los profesionales en salud sobre la mejor fórmula para la medición de la TFG en edades avanzadas, así como la importancia de saber interpretar resultados y validar las diferentes fórmulas según la población en la que se apliquen.

De las fórmulas disponibles más utilizadas en la práctica clínica actual, la ecuación CKD- EPI es la recomendada por las últimas guías Kidney Disease Improving Global Outcomes (KDIGO) del 2012 para estimar el filtrado glomerular, esta fórmula aumenta su sensibilidad cuando se utiliza la combinación de marcadores endógenos como la creatinina y la cistatina $\mathrm{C}$.

\section{Los autores declaran no tener conflicto de interés.}

\section{REFERENCIAS}

1. Musso CG, Álvarez-Gregori J, Jauregui J, MacíasNúñez JF. Glomerular filtration rate equations: a comprehensive review. Int Urol Nephrol [Internet]. 2016;48(7):1105-10. Disponible en: http://dx.doi.org/10.1007/s11255-016-1276-1

2. Raman M, Middleton RJ, Kalra PA, Green D. Estimating renal function in old people: an in-depth review. Int Urol Nephrol [Internet]. 2017;49(11):1979-88. Disponible en: http://dx.doi.org/10.1007/s11255-017-1682-z

3. Levey AS, Inker LA. Assessment of glomerular filtration rate in health and disease: A state of the art review: State of the art review for clinical pharmacology and therapeutics. Clin Pharmacol Ther [Internet]. 2017;102(3):405-19. Disponible en: http://dx.doi.org/10.1002/cpt.729

4. Musso CG, de Los Rios E, Vilas M, Terrasa S, Bratti G, Varela F, et al. The HUGE formula (hematocrit, urea, gender) for screening for chronic kidney disease in elderly patients: a study of diagnostic accuracy. Int Urol Nephrol [Internet]. 2017;49(4):677-80. Disponible en: http://dx.doi.org/10.1007/s11255-016-1486-6

5. Zou L-X, Sun L, Nicholas SB, Lu Y, K SS, Hua R. Comparison of bias and accuracy using cystatin $C$ and creatinine in CKD-EPI equations for GFR estimation. Eur $\mathrm{J}$ Intern Med [Internet]. 
2020;80:29-34. Disponible en: http://dx.doi.org/10.1016/j.ejim.2020.04.044

6. Wang Y, Levey AS, Inker LA, Jessani S, Bux R, Samad $Z$, et al. Performance and determinants of serum creatinine and cystatin C-based GFR estimating equations in South Asians. Kidney Int Rep [Internet]. 2021;6(4):962-75. Disponible en: http://dx.doi.org/10.1016/j.ekir.2021.01.005

7. Heras M, Fernández-Reyes MJ. Nuevas herramientas para abordar la función renal en ancianos: la ecuación Berlin Initiative Study y la fórmula hematocrito, urea y género. Med Clin (Barc) [Internet]. 2016;146(10):450-4. Disponible en: http://dx.doi.org/10.1016/j.medcli.2016.01.012

8. Freidin N, O'Hare AM, Wong SPY. Personcentered care for older adults with kidney disease: Core Curriculum 2019. Am J Kidney Dis [Internet]. 2019;74(3):407-16. Disponible en: http://dx.doi.org/10.1053/j.ajkd.2019.01.038

9. Bargnoux A-S, Barguil Y, Cavalier E, Cristol J-P. Estimation of glomerular filtration rate using cystatin C. Ann Biol Clin (Paris) [Internet]. 2019;77(4):375-80. Disponible en: http://dx.doi.org/10.1684/abc.2019.1456

10. Pasala S, Carmody JB. How to use... serum creatinine, cystatin C and GFR. Arch Dis Child Educ Pract Ed [Internet]. 2017;102(1):37-43. Disponible http://dx.doi.org/10.1136/archdischild-2016$\underline{311062}$

11. Griffin BR, Liu KD, Teixeira JP. Critical care nephrology: Core Curriculum 2020. Am J Kidney Dis [Internet]. 2020;75(3):435-52. Disponible en: http://dx.doi.org/10.1053/i.ajkd.2019.10.010

12. Inker LA, Tighiouart $\mathrm{H}$, Coresh J, Foster MC, Anderson $\mathrm{AH}$, Beck GJ, et al. GFR estimation using $\beta$-trace protein and $\beta 2$-microglobulin in CKD. Am J Kidney Dis [Internet]. 2016;67(1):408. Disponible en: http://dx.doi.org/10.1053/j.ajkd.2015.07.025

13. Li Z, Wang Z. Aging kidney and aging-related disease. Adv Exp Med Biol [Internet]. 2018;1086:169-87. Disponible en: http://dx.doi.org/10.1007/978-981-13-1117-8 11

14. Moore PK, Hsu RK, Liu KD. Management of acute kidney injury: Core curriculum 2018. Am J Kidney Dis [Internet]. 2018;72(1):136-48. Disponible en: http://dx.doi.org/10.1053/j.ajkd.2017.11.021

15. Mora-Gutiérrez JM, Slon Roblero MF, Castaño Bilbao I, Izquierdo Bautista D, Arteaga Coloma J, Martínez Velilla N. Rev Esp Geriatr Gerontol [Internet]. 2017;52(3):152-8. Disponible en: http://dx.doi.org/10.1016/j.regg.2016.03.006 